\title{
ON THE NUMBER OF ELEMENTS OF A GROUP WHICH HAVE A POWER IN A GIVEN CONJUGATE SET*
}

BY LOUIS WEISNER

1. Introduction. A fundamental theorem on abstract groups is Frobenius' theorem: The number of elements in a group of order $g$ whose $n$th powers belong to a given conjugate set is zero or a multiple of the greatest common divisor of $g$ and $n$. In this paper, I will prove the following theorems, which are also concerned with the number of elements having a power in a given conjugate set.

TheORem 1. The number of elements of a group whose nth powers are in a given conjugate set is either zero, or a multiple of the number of elements in the conjugate set.

THeOREM 2. In a group of order $g$, the number of elements which have a power in a given conjugate set of elements of order $n$ is a multiple of the greatest divisor of $g$ that is prime to $n$.

An interesting deduction from Theorem 2 is the following theorem.

THEOREM 3. In a group of order $g$, the number of elements whose orders are multiples of $n$ is either zero, or a multiple of the greatest divisor of $g$ that is prime to $n$.

2. Proof of Theorem 1 . Let $t_{1}, t_{2}, \cdots, t_{x}$ be the elements of a group $G$ which satisfy the equation $t^{n}=s_{1}$, and let the conjugates of $s_{1}$ under $G$ be $s_{1}, s_{2}, \ldots, s_{m}$. There exist elements $u_{1}, u_{2}, \cdots, u_{m}$ in $G$ such that

Since $t_{a}^{n}=s_{1}$

$$
u_{i}^{-1} s u_{i}=s_{i}, \quad(i=1,2, \ldots, m) .
$$

$$
\left(u_{i}^{-1} t_{a} u_{i}\right)^{n}=u_{i}^{-1} s u=s_{i} .
$$

\footnotetext{
* Presented to the Society, February 28, 1925.
} 
Hence there are exactly $x$ elements of $G$ whose $n$th powers are $s_{i}$. If $u_{i}^{-1} t_{a} u_{i}=u_{k}^{-1} t_{b} u_{k}$, then, raising both members to the $n$th power, we have

$$
u_{i}^{-1} s u_{i}=u_{k}^{-1} s u_{k},
$$

or $s_{i}=s_{k}$, whence $i=k$. It follows that $t_{a}=t_{b}$, whence $a=b$.

The distinct elements of $G$ whose $n$th powers are conjugate to $s_{1}$ are therefore

and their number is $m x$.

$$
u_{i}^{-1} t_{a} u_{i}, \quad\left(\begin{array}{c}
a=1,2, \cdots, x \\
i=1,2, \cdots, m
\end{array}\right),
$$

3. Proof of Theorem 2. Suppose, first, that the conjugate set consists of only one element $s$, which is therefore invariant under the group $G$. Let $k$ be the greatest divisor of $g$ that is prime to $n$; and let $t^{a}=s$.

CASE 1: a prime to $n$. If $a a^{\prime} \equiv 1(\bmod n)$, then $t=s^{a \prime}$. An element $u$ of $G$ of order $m$ prime to $n$, being commutative with $s$, is commutative with $t$. Hence

$$
(t u)^{x m}=s^{a \prime x m} u^{x m}=s,
$$

where $x a^{\prime} m \equiv 1(\bmod n)$. Hence $s$ is a power of tu.

By Frobenius' theorem, $G$ contains $\lambda k$ ( $\lambda$ integral) elements whose orders divide $k$. Denote these elements by $u_{1}, \ldots, u_{\lambda}$. We have just proved that the only elements of $G$ satisfying the equation $t^{a}=s$, where $a$ assumes all values prime $n$, are

$$
s^{c} \mu_{z}, \quad\left(\begin{array}{l}
\mu=1, \cdots, \varphi(n) \\
z=1, \cdots, k
\end{array}\right),
$$

where $c_{1}, \ldots, c_{\varphi(n)}$ are the integers not greater than $n$ and prime to $n$. These elements are distinct; ${ }^{*}$ hence their number is a multiple of $k$.

CASE 2: a not prime to $n$. Let $a=n_{1} b$, where $b$ is the greatest divisor of $a$ that is prime to $n$. We may write $t=t_{1} t_{2}$, $\dagger$ where $t_{1}$ and $t_{2}$ are powers of $t$, and the order of $t_{2}$ is the greatest divisor of the order of $t$ that is prime to $n$, while the order of $t_{1}$ is a multiple $n_{2}$ of $n$ which is not divisible by a number prime to $n$ (except

* W. Burnside, Theory of Groups, 1911, § 16.

$\dagger$ Burnside, loc. cit. 
unity). If the order of $t_{2}$ is $c$, then the order of $t$ is $n_{2} c$. Since $s=t^{n_{1} b}=t_{1}^{n_{1} b} t_{2}^{n_{1} b}$, we may write

whence

$$
s^{n}=t_{1}^{m n_{1} b} t_{2}^{m n_{1} b}=1,
$$

$$
t_{1}^{n n_{1} b}=t_{2}^{n n_{1} b}=1 \text {. }
$$

Hence $c$ is a divisor of $n n_{1} b$ and therefore of $b$. It follows from (2) that $s=t_{1}^{n_{1} b}$. If $u$, of order $m$ prime to $n$, is commutative with $t$, and if $m^{\prime} m \equiv 1\left(\bmod n_{2}\right)$, then

$$
\left(t_{1} u\right)^{m^{\prime} m m_{1} b}=t_{1}^{m^{\prime} m n_{1} b} u^{m^{\prime} m n_{1} b}=t_{1}^{n_{1} b}=s .
$$

Hence $s$ is a power of $t_{\mathrm{r}} u$.

Let $n_{3} k_{1}$ be the order of the normaliser $N$ of $t$ in $G$ and let $g / n_{3} k_{1}=n_{4} k_{2}$, where $k_{1}$ and $k_{2}$ are the greatest divisors of $n_{3} k_{1}$ and $n_{4} k_{2}$ respectively that are prime to $n$ and hence to $n_{2}$. The number of elements of $N$ whose orders are prime to $n$ is of the form $\alpha k_{1}$; denote these elements by

$$
u_{1}, \cdots, u_{\alpha k_{1}} \text {. }
$$

It follows from (3) that $s$ is a power of

$$
t_{1} u_{1}, \cdots, t_{1} u_{\alpha k_{1}} \text {. }
$$

It is noteworthy that $t_{2}$ is in (4) and hence $t=t_{1} t_{2}$ is in (5). Let $t_{1}^{\prime}=w^{-1} t_{1} w$ be a conjugate of $t_{1}$. Since $s$ is invariant under $G$,

$$
t_{1}^{\prime n_{1} b}=w^{-1} t_{1}^{n_{1} b} w=w^{-1} s w=s .
$$

Now there are exactly $\alpha k_{1}$ elements in $G$ whose orders are prime to $n$ and which are commutative with $t_{1}^{\prime}$. Denoting these by $u_{1}^{\prime}, \ldots, u_{\alpha k_{1}}^{\prime}$, it follows that $s$ is a power of

$$
t_{1}^{\prime} u_{1}^{\prime}, \ldots, t_{1}^{\prime} u_{c k k_{1}}^{\prime} \text {. }
$$

Moreover, no element in (6) is equal to an element in (5).* There being $n_{4} k_{2}$ conjugates of $t_{1}$, we obtain $\alpha n_{4} k_{1} k_{2}$ elements of which $s$ is a power. Observing that $k=k_{1} k_{2}$ is the greatest divisor of $g$ prime to $n$, it follows that $t_{1}$ and its conjugates give rise in the manner described above to a

* Burnside, loc. cit. 
multiple of $k$ elements of which $s$ is a power. These elements are evidently distinct from those obtained under Case 1 .

If $s$ is a power of $\tau$ and $\tau$ is not one of the elements already obtained, let $\boldsymbol{\tau}=\boldsymbol{\tau}_{1} \tau_{2}$, where the order of $\boldsymbol{\tau}_{2}$ is the greatest divisor of the order of $\tau$ that is prime to $n$. Then $\tau_{1}$ and its conjugates give rise to a multiple of $k$ elements of which $s$ is a power. Let $\tau_{1} v$ be one of these elements, and, if possible, let it be equal to an element in (5), say $\tau_{1} v=t_{1} u=v$. Since the order of $u$ and the order of $v$ are both equal to the greatest divisor of the order of $w$ that is prime to $n$, we must have $\tau_{1}=t_{1}, v=u$. This is not the case, and hence $\tau_{1}$ and its conjugates give rise to a multiple of $k$ new elements of which $s$ is a power.

The theorem now follows under the assumption that $s$ is invariant under $G$.

Suppose next that the conjugate set consists of powers of $s$, so that $(s)$ is invariant under $G$. Denote the conjugates of $s$ by

$$
s^{c_{1}}, \ldots, s^{c_{r}}, \quad\left(c_{1}=1\right),(r>1) .
$$

Let $H$, of order $h$, be the normaliser of $s$ in $G$; and let $k$ be the greatest divisor of $h$ that is prime to $n$. The number of elements in $H$ of which $s$ is a power is a multiple of $k$, which we denote by $\lambda k$. Since $c_{1}, \ldots, c_{r}$ are prime to $n, s^{c_{1}}, \ldots, s^{c_{r}}$ are powers of these same $\lambda k$ elements. Hence $H$ contains exactly $\lambda k$ elements which have a power in (7). If $t$ has a power in (7), so has $t^{c_{i}}(i=1,2, \cdots, r)$. Hence the number of elements of $G$ which have powers in (7) is a multiple of $r$. The order of $G$ is $g=r h$; for $G / H$ is simply isomorphic with the group obtained by establishing an isomorphism of $s$ with $s^{c_{1}}, \ldots, s^{c_{r}}$, and is of order $r$. The number of elements of $G$ which have powers in (7) is a multiple of $r$ and a multiple of $k$ and is therefore a multiple of the greatest divisor of $g$ that is prime to $n$.

Finally, suppose the conjugate set of elements does not consist of the powers of one of them. The elements in the conjugate set may be separated into subsets 


$$
\begin{aligned}
& s_{1}^{c_{1}}, \ldots, s_{1}^{c_{r}}, \quad\left(c_{1}=1\right), \\
& s_{2}^{c_{1}}, \ldots, s_{2}^{c_{r}}, \quad(r \geqq 1), \\
& \ldots \ldots \ldots, \ldots \ldots \ldots \cdots \cdots
\end{aligned}
$$

such that any two elements in the same subset are powers of each other, whereas no element is a power of an element in another subset.

Let $H_{i}$, of order $h$, be the normaliser of $\left(s_{i}\right),(i=1, \cdots, m)$. An element of $G$ which has a power in $\left(8_{i}\right)$ is commutative with the elements of $\left(8_{i}\right)$ and hence is in $H_{i}$. Therefore $H_{i}$ contains all the elements of $G$ which have powers in $\left(8_{i}\right)$. Since the elements of $\left(8_{i}\right)$ form a complete set of conjugates under $H_{i}$, the number of elements of $G$ which have powers in $\left(8_{i}\right)$ is $\lambda k$, where $\lambda$ is an integer, and $k$ is the greatest divisor of $h$ that is prime to $n$. If $t$ has a power in $\left(8_{i}\right), t$ cannot have a power in $\left(8_{j}\right),(i \neq j)$. For if $t^{x}=s_{i}^{c}$ and $t^{y}=s_{j}^{c^{\prime}}$, then $t^{x}$ and $t^{y}$ are of the same order $n$, so that each is a power of the other, whence $i=j$. It follows that the number of elements of $G$ which have a power in (8) is $\lambda \mathrm{kg} / h$, and this number is evidently a multiple of the greatest divisor of $g$ that is prime to $n$.

4. Proof of Theorem 3. If the group $G$ contains an element of order $n$, separate the elements of order $n$ into complete sets of conjugates, which we denote by $C_{1}, \ldots, C_{x}$. Of these, we select a subset $C_{1}, \ldots, C_{y}$, such that no element in $C_{i}$ has a power in $C_{j}(i \neq j),(i, j=1, \cdots, y)$. Every element of $G$ whose order is a multiple of $n$ has a power in one and only one of the sets $C_{1}, \ldots, C_{y}$; and the order of every element which has a power in one of these sets is a multiple of $n$. Since the number of elements of $G$ which have a power in $C_{i}$ is a multiple of the greatest divisor of $g$ that is prime to $n$, it follows that the number of elements of $G$ whose orders are multiples of $n$ is a multiple of the greatest divisor of $g$ that is prime to $n$. 